\title{
On the Design Thinking and Modeling Method of Animation Roles
}

\author{
Liangwei Lv \\ Yantai Nanshan University \\ Yantai, China
}

\begin{abstract}
The image of animation roles shall be designed according to the requirements of script, taking the overall artistic conception on film by the director into consideration, making the character modeling not only have the nature of integrality, but also highlighting the details, reflecting the character traits in terms of modeling. The mental activity of the characters shall also be described, giving the individualized characteristics and typical language and movements to the characters, enabling the audiences to remember them for a long time.
\end{abstract}

Keywords—animation roles; design thinking; modeling design

\section{INTRODUCTION}

Image design of animation roles is an essential part for the animation film, playing a very important role. While designing the character image, first of all the design thinking shall be cleared, and then to shape the character image on the basis of basic principle of character design. In shaping the character image, the basic form of the characters and the head design are the key parts of character modeling.

\section{THINKING OF ANIMATED CHARACTER DESIGN}

Upon entering into the phase of character design, first of all, the character modeling shall be designed according to script requirements. The formal features and modeling style shall be taken into consideration while designing, and every aspects of the animated film described by the script shall be analyzed carefully, obtaining the times, historical background, character features (gender, nationality, age, profession, characteristics, appearance, etc.), relationship among the characters, character movements, clothing and props from the script, and indicate clearly the style characteristics of the character modeling, the space where characters exist, individualized characteristics of the characters and typical movements of the characters by virtue of the contents in the script. And then conduct the finishing process for the data to obtain general outline of the characters. Deepen the characteristics of every part of the character image by discussion, making the character personality unique, distinct and vivid which has emotion and feelings, being able to catch audiences' emotion, leaving profound impression to the audiences in terms of their gestures and expressions. For example, the characters in the animation film Hua Mulan are granted with different modeling and characteristics according to requirements of the scripts. As a girl, Mulan disguised as a boy and joined the army for her father. The representative of Chinese Dragon and the strong Huns are all designed according to the story line.

Secondly, the director's overall artistic thinking of the film shall be taken into consideration and grasp the modeling characteristics of the characters, making the character modeling not only have the nature of integrality, but also highlighting the details, reflecting the character traits in terms of modeling. The mental activity of the characters shall also be described, giving the individualized characteristics, typical language and movements to the characters, enabling the audiences to remember them for a long time. While designing the animation roles, large amount of materials are needed to be collected. Observe the details in life carefully and dig traditional culture in detail, observing the familiar things appear in life, such as people, animals, clothing and natural environment closely, to accumulate abundant modeling design data. No matter what type of character you are designing, the story line shall be determined first of all, thus the in-depth understanding and investigation may be conducted against story background. For example, large amount of data such as historical background, clothing, nationality characteristics and regional culture are all collected for the story of Hua Mulan, to act as design reference after data summary has been conducted. And while designing the characters of Kung Fu Panda and Finding Nemo, large amount of animal prototypes have been collected, indicating fully that without accumulation of materials, there won't be good creation inspiration for character image design.

\section{POSITIONING OF ANIMATED CHARACTER DESIGN}

At the beginning of animated character design, the positioning of character image is very important, which is the starting point for the designer to expand design thinking. A successful animation work must be of a certain type of style and characteristics, and only by this, can it attracts audiences, rendering the work long lasting vitality. And the character image with distinct characteristics can only be shaped out by completely relying on the comprehensive positioning of the character image. Character design in the animation shall be positioned from the following aspects:

Character design of animation shall be positioned in according to its audiences. While conducting the character design, first of all, the discussion with director and script composer shall be conducted to determine the audiences of the animation; then dig the character features according to the 
script, as different audiences have different value concept and aesthetic standard, and they will generate different mental response. The purpose of the characters designed is to move the audiences, thus to make them be attracted by the characters designed by us, enabling the animation roles be more popular.

The audiences can be divided by ages, regions, culture and social class, thus different design positioning can be formed. For example, as for the animation works against children, designers shall consider that the audiences at this age phase are generally keen on the animation roles that are simple and straightforward with distinct characteristics while designing, and consider that characters with strong affinity may easily shorten the distance with children, such as the animations of Chibi Maruko-Chan and SpongeBob SquarePants. And as for the animation works against senior and high school students, while designing the character modeling, certain aesthetic standard of the teenagers at this age phase shall be considered, as they have their own appreciation orientation. Such kind of audiences are generally love affection films and those with interesting story line, for example the animations of The Prince of Tennis and Naruto.

While designing the animation roles, the positioning shall be made by taking into consideration the media forms. Different media forms will have different expression forms and characteristics, restricting the production of animation to a certain degree. Different media have their own broadcasting characteristics, for instance, film, television, network and mobile phone are all carrying animations, but the animations broadcasted by different media are different in terms of expression form, theme, production methods and character design style. Film animation shall catch the audiences' inner thoughts within restricted time, which requires the film animation to highlight theme, with shocking picture effect and distinct character image characteristics. The television animation is broadcasted episode by episode, and each episode has an independent story line. Characters in such animation can exist before the audiences for a long time. Although the characters are simple and generalized, they have left a profound impression to the audiences. Network and mobile phone are the new media appeared recently with characteristics of init iative and interactivity. The animated character design of such media shall be of selectivity and of certain timeliness, for example The Pobaby.

Character Personality Positioning. The so-called personality positioning refers to the characteristic determination of characters to be designed, which is the same as individualized process of actors to play certain roles in a film. In the film, each character has its own characteristics. The actors' appearances shall be dressed up, their language shall be enriched and the emotion treatment shall be strengthened according to those characteristics, so dose the individualize process of animation roles. Determine their characteristics according to requirements of story line, then conduct relevant factor positioning process, which includes: body shape, skin color, expression description, clothing and movement expression and so on. Only by grasping the colorful character positioning in animation, can the details of every character be shaped perfectly.

\section{THE BASIC CRITERIA FOR THE DESIGN OF ANIMATION} ROLES

The basic criteria of animation roles design mainly include contrast and harmony, rhythm and unity.

\section{A. Contrast and harmony}

Contrast refers to the comparison of roles in shape, style, color, picture composition and others during the role design. Contract is purposed to well reflect the distinct features of role and make the role image full of rich visual effect. Contract includes the modeling contract of role itself and the modeling features contract among roles, of which the former includes contract of shape structure, contract of partial shape and contract of lines, and the later includes contract of proportional among roles, contract of modeling structure and contract of individual features. So we can design unique images by using these contrast methods of role modeling, to make them more attractive.

Harmony refers to the unified coordination of the role image modeling styles, the harmonization of color arrangement, the harmonization of formal performance, and the unified coordination among roles, making the role model in the film have more unification in form.

\section{B. Cadence and unity}

Cadence refers to the sense of rhythm appearing in the works of art. In the modeling of animation roles, the size, repeat and change of shape and the combination of different shapes will give us a different feeling. Everything in the objective world has its own sense of rhythm in shape, so we can make great exaggeration of orig inal shape in our modeling design to strengthen the existing sense of rhythm. We should make the slow and delicate rhythm of prototype more intense and bouncing and make the image more varied and full of sense of rhythm.

Unity refers to the integrity of role image in modeling. In the design of role image, if we make it too loose, scattered and disorderly in modeling, we will be unable to keep the integrity of role image, but if we only emphasize the local details, we will weaken the expressive force of role image, so that we will loss the overall artistic effect of role image.

\section{THE DESIGN PROCESS OF ANIMATION ROLES}

Basic shape design. When designing the basic shape design of role, we should first determine the body proportion. Usually, we will calculate the body proportion as per head as unit, while the proportion of the body is decided by gender, age and ethnic group, and the length of body should be that of 7-8 heads. In the design of role, we also should make the body proportion relationship of role according to the characteristics of the role. In order to enhance the dramatic expression of role, we must highlight the facial expressions of role, so we must change the proportion between head and body, reduce the proportion of body to make the visual characteristics of role more obvious. Second, we must make basic figure shaping of role. We should outline the external modal structure of role according to the needs or fit several basic geometric shapes together, to reflect 
the main features of role and show the figure of the whole role and further to constitute the basic shape roles. In the basic design, we should make it reflect the contrast effect among the roles, and grasp the structural features of role, to deliver the basic shape of role with simple lines and the technique of exaggeration and generalization, so that the basic shape is more suitable for the foundation of role modeling.

Head design. In animation role design, the head of character is particularly important in design. It is because the head design could reflect the age, traits, expression and other typical characteristics. The head is of the most visual impact and performance, and the head is the focus of attention of the audience. Head design is divided into three parts: facial design, facial features design and hair design.

Hair design. The hair style of character could pass information, like appearance, character, occupation and etc. The hair design shall be consistent with the traits of character, face, identity and temperament.

Facial design. Due to the impact of age, gender, race and figure, shapes of face are also different from each other. And the facial design of character is varied. The shape of face can be summed up as oval, round and square. And then depict facial features of character in detail according to design requirements. For example, some old people are of thin and long face, and some are of round and fleshy face; s ome are of small forehead with wrinkles. So me of them are with no tooth, and thus the mouth is concave inwardly. Most children are of round and oblate face, with big forehead and short and small jaw.

Facial features design. Facial features design mainly refers to the modeling of eyes, eyebrows, nose, mouth and ears. Designers can sum up facial features for corresponding character according to the basic shape of human facial features. For example, in My Neighbor Totoro, a little sister of four or five years old has two braids, two curious big eyes, shining with light. Her mouth is big, and she often laughs a hearty laugh. In animation role modeling, designers often make eye modeled, and simply summarize an eye effect in line with the expression of character in order to conform to the animation style. The character's nose is exaggerated and processed simply, so that the nose has a certain representation. The importance of mouth is just behind the eyes among facial features. Mainly use lines and geometries to depict mouth and make mouth design according to basic forms of human's mouth in order to express different body features. In the animation, the form of the mouth is different in size, length and position. Sometimes it can be simple and sometimes it can be exaggerated. The form of mouth is to serve the expression of character. Simply use the summarized method to depict the ears, and use lines to express helix, antihelix and tragus. And then design shape of ears according to head style of character.

Costumes of characters. Clothing style structure, color collocation and fabric treatment can effectively portray out character's social status, occupation classification, cultural level, life habits, personality, hobbies, ethnic areas, etc. At the same time, clothing has the role of symbol and identification. According to the needs of the plot, clothing can be divided into different ethnic costumes, different historical costumes, different regional costumes, different space-time fantasy costumes and fashionable costumes. Lovely girls often wear clothes of Lolita style, such as round puff-sleeved clothes, pleated skirt with floral lace, and shoes with round toe cap. It gives people a lovely pleasant feeling. Sexy girls often wear tights, fully showing the S-shape figure, and occasionally expose a little of lace underwear. They wear high-heeled shoes, increasing the charm of women. Same woman is shaped into completely different characters due to worn costumes. It needs to analyze and experience the difference carefully. Stable middle-aged men mainly wear business suit with a necktie and black leather shoes, and carry a data bag or laptop bag. Stable young men often wear plainly and simply, like a student. Boys pursuing fashion are often cool, and of punk style. They like wear various small ornaments.

In the vast majority of cases, the animation roles are in shoes in the performance in the film. Therefore, it is very necessary to master the basic expression law of shoes in design and performance. In animation film, we divide shoe into two parts, heel and sole (including sole and toes), for design. In depicting the shoes of character, pay attention to perspective state of character, for the trend and the direction of the shoe sole and the shoe vamp directly reflect the perspective relation between character and environment. In drawing, it is important to establish a basic concept of three-dimensionally depicting shoes in order to avoid forceless or scattered shoes.

Props design. In the animation, characters often wear or carry a variety of portable props. These props have a very close relationship with characters, and they can strengthen the traits and figure features of characters, and show identity, status, interests and hobbies of characters. They effectively heighten the character and increase the appeal of character. There are a variety of portable props for character, including daily necessities, artworks, knife and sword, as well as some magic implements to perform magic arts.

\section{CONCLUSION}

In summary, while designing character image, first of all, the script requirements shall be followed, and secondly, director's overall artistic thinking on the film shall be taken into consideration, and then conduct character image modeling in accordance with the basic principle of character design. During the process of shaping the characters, emphasis shall be laid on basic form and head design, making the character image in line with requirements of animation film. Only the animated character image has undergone character design positioning, design material collection, character image thinking and drawing, can it be an animated character image widely known by people. It can be easily seen that the creation process of animated character image is reasonable and in order, meanwhile, it will directly influence the establishment of character image. As a result, we shall grasp the creation process of character image, and pay attention to the role of every segment. 


\section{REFERENCES}

[1] Li Tie, Zhang Haili. Animation Roles Design. Beijing Jiaotong University Press.

[2] Wu Guanying. Animation Modeling Design. Tsinghua University Press.

[3] Chen Hailu. Animation Roles Design. Shanghai People's Fine Arts Publishing House.

[4] Chen Guo, Hong Fang. Thought and Skills of Animation roles Modeling. Communication University of China Press. 